\title{
Incidental lung findings in coronary computed tomography angiography
}

\author{
Tuğba Eldeş ${ }^{1}$, Bilge Yilmaz Kara²*
}

\section{SUMMARY}

OBJECTIVE: In coronary computed tomography angiography, a part of the lung parenchyma also enters the image area which is called the field of view. The aim of this study was to evaluate the rate of pulmonary abnormalities and document their association with demographic features in subjects undergoing multislice coronary computed tomography angiography obtained for the assessment of coronary artery disease.

METHODS: This was a retrospective observational study evaluating the coronary computed tomography angiography scans of 1,050

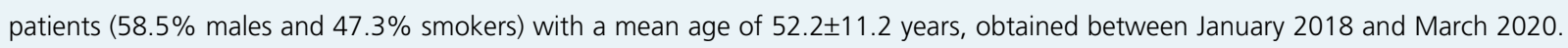
Pulmonary abnormalities were reported as nodules, focal consolidations, ground-glass opacities, consolidations, emphysema, cysts, bronchiectasis, atelectasis, and miscellaneous.

RESULTS: In total, 274 pulmonary abnormalities were detected in 266 patients (25.3\%). The distribution of incidental lung findings was as follows: pulmonary nodules: $36.4 \%$, emphysema: $15.6 \%$, bronchiectasis: $11 \%$, ground-glass opacities: $7.2 \%$, atelectasis $7.2 \%$, focal consolidations: $5 \%$, cysts: $6 \%$, consolidations: $2.5 \%$, and miscellaneous: $9.1 \%$. The patients with pulmonary pathology were older ( $55.5 \pm 11.4$ versus $51.0 \pm 10.9$ years), and the percentage of smokers was higher (60.1 versus $43.2 \%)$. The possibility of the presence of any incidental lung findings in field of view of coronary computed tomography angiography increases significantly over the age of 40.5 years ( $p<0.001$, AUC 0.612, 95\%Cl 0.573-0.651).

CONCLUSION: Multislice coronary computed tomography angiography can give important clues regarding pulmonary diseases. It is essential for the reporting radiologist to review the entire scan for pulmonary pathological findings especially in patients with smoking history and over the age of 40.5 years.

KEYWORDS: Computed tomography angiography. Solitary pulmonary nodule. Incidental findings.

\section{INTRODUCTION}

Coronary artery disease $(\mathrm{CAD})$ maintains its high-risk potential for morbidity and mortality in the stressful life pace of our age. Coronary computed tomography angiography (CCTA), which is a non-invasive technique that can display the anatomical structure and features of the vascular lumens of the coronary arteries along with the hilar-perihilar lung parenchyma within the FOV, is an important non-invasive tool with excellent negative predictive value (99\%) ${ }^{1}$. Additionally, some of the risk factors for $\mathrm{CAD}$, such as increasing age and smoking, are also risk factors for pulmonary pathological findings such as bronchial carcinoma ${ }^{2,3}$.

The issue of incidental imaging findings, in patients undergoing imaging for an unrelated reason, is the subject of debate. First of all, these findings may lead to anxiety in patients. Because of the clinical uncertainty regarding their

'Recep Tayyip Erdoğan University, Department of Radiology - Rize, Turkey.

${ }^{2}$ Recep Tayyip Erdoğan University, Department of Pulmonary Medicine - Rize, Turkey.

*Corresponding author: drbilgeyilmaz@hotmail.com

Conflicts of interest: the authors declare there are no conflicts of interest. Funding: none.

Received on July 14, 2021. Accepted on August 03, 2021. 
ideal management, they frequently lead potential over investigations. In addition, it is difficult for clinicians to appropriately inform patients of the chance of incidental findings because the prevalence is inconsistent and unclear ${ }^{4}$. Similarly, there are contradictory opinions about the benefits of scanning and reporting lung fields included in the CCTA 5 . The aim of this study was to provide information about the incidental lung findings in the FOV of patients undergoing routine CCTA and to interpret the results in a way that is more practical for medical application.

\section{METHODS}

This study was designed as an observational, retrospective, cross-sectional study, and the data were obtained from the patients' records between January 2015 and December 2019. Lesions in the lung parenchyma and the hilar-mediastinal regions that entered the FOV of routine CCTA scans were recorded. We searched for the available data related to smoking and identified the participants as smokers if they were current smokers or ex-smokers with a smoking history of more than 20 pack-years. This study was approved by the Recep Tayyip Erdogan University Clinical Researches Ethical Committee (No.: 2019/162).

\section{Coronary CT angiography procedure}

All CCTA scans were performed after oral $\beta$-adrenergic receptor blocker administration $12 \mathrm{~h}$ before the procedure. The blood pressure and the heart rate were monitored at 5-min intervals by an experienced cardiologist ${ }^{6}$. Region of interest was placed in the aorta that emerged with the bolus tracking technique ${ }^{7}$, and scanning was started automatically when the contrast density reached $300 \mathrm{HU}$.

All patients were referred to have a radiologic assessment and have been retrospectively selected regardless of their pre-diagnosis. The CCTA scans have been run in different phases according to the electrocardiography triggering method ${ }^{8}$ with 128 detectors (General Electric Discovery CT750 HD CT device). All CCTA images were evaluated by a radiologist with a national cardiovascular certificate of competence.

\section{Assessment of pulmonary findings}

Patients who were under the age of 18 years and who had a bronchial carcinoma history or lung surgery, such as lobectomy or pneumonectomy, and those with missing data and consecutive CCTA recordings were excluded from this study. The incidental lung findings were grouped under the following headings: solid nodule, calcified nodule, ground glass nodule, focal consolidation, ground-glass opacity (GGO), consolidation, emphysema, cyst, bronchiectasis, atelectasis, and miscellaneous (pleural effusion, pleural calcification, etc.)

\section{Statistical analysis}

SPSS 20.0 software (SPSS Inc., Chicago, USA) was used for statistical analysis. Parametric data were presented as mean \pm SD. The normality test was performed on all variables. The Student's $t$-test was used for parametric variables, and Mann-Whitney $\mathrm{U}$ test was used for the nonparametric distribution of variables. Spearman's and Pearson's tests were used for correlation analysis. Yates correction, chi-square test, and Fisher's exact test were used for the comparison of categorical data.

Receiver operating characteristic (ROC) curve analysis was used to find out the cutoff value, sensitivity, and specificity of age for predicting the presence of pulmonary pathology. Differences were considered statistically significant if the $\mathrm{p}<0.05$.

\section{RESULTS}

The CCTA scans of 1,050 patients (58.5\% males and $47.3 \%$ smokers) were retrospectively evaluated. The mean age was $52.2 \pm 11.2$ (minimum-maximum: $19-89$ ) years. A total of 274 incidental lung findings were present in 266 patients (25.3\%). The rates of pathological findings were as follows: nodules: 100 (solid nodules: 85 , ground-glass nodules: 10 , and calcified nodules: 5), emphysema: 43 , bronchiectasis: 30 , miscellaneous (pleural effusion, pleural thickening, etc.): 25 , atelectasis: 20 , GGOs: 20, cysts: 16, focal consolidation: 13, and consolidation: 7 (Figure 1). The number of the pathological pulmonary findings according to localization was as follows: left upper lobe: 14, lingula: 34 , left lower lobe: 59 , right upper lobe: 23 , right middle lobe: 48 , right lower lobe: 25 , diffuse: 70 , and miscellaneous (pleura, fissures, etc.): 20 lesions. A case of bronchial carcinoma, a case of esophageal cancer, a giant sliding hernia, and a foreign body in the right lower lobe bronchus were also reported (Figure 2).

When patients with and without pulmonary pathology were compared, those with pulmonary pathology were older and the percentage of smokers was higher (Table 1).

Advanced age and smoking have been found to positively correlate with the presence of pulmonary pathology. In the ROC analysis, the cutoff value of age for predicting the presence of pulmonary pathology was 40.5 years $(\mathrm{p}<0.001$, AUC 0.612, 95\%CI 0.573-0.651, sensitivity: 91.4\%, specificity: $17.1 \%$, negative predictive value: $94 \%$, positive predictive value: $11 \%$, and positive and negative likelihood ratio: 1.1 and 0.53 , respectively). 


\section{Percentage of Pulmonary Pathologies}
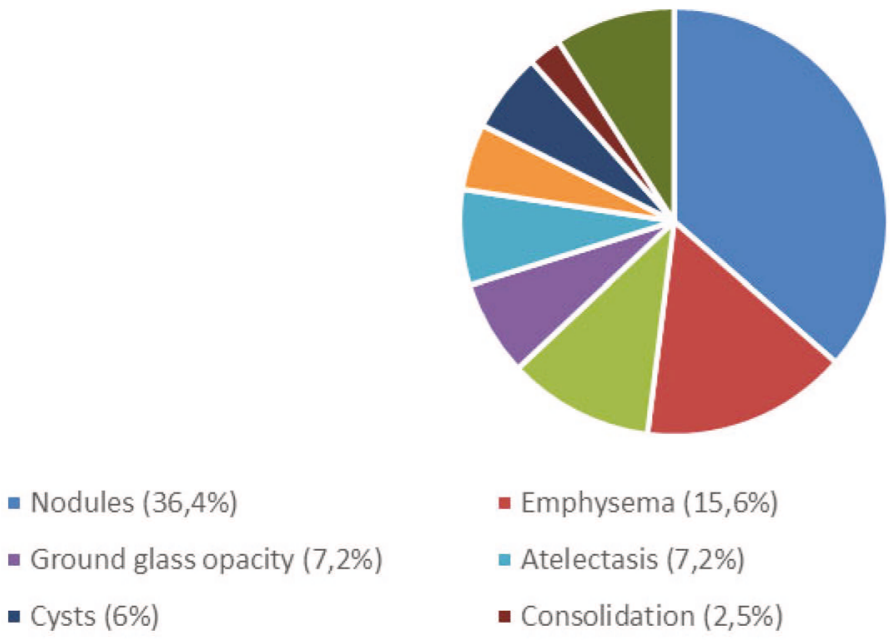
- Emphysema (15,6\%)
- Atelectasis $(7,2 \%)$
- Consolidation $(2,5 \%)$

\section{- Bronchiectasis (11\%) \\ - Focal consolidation (5\%) \\ - Miscellaneous $(9,1 \%)$}

Figure 1. Types of pulmonary pathologies.
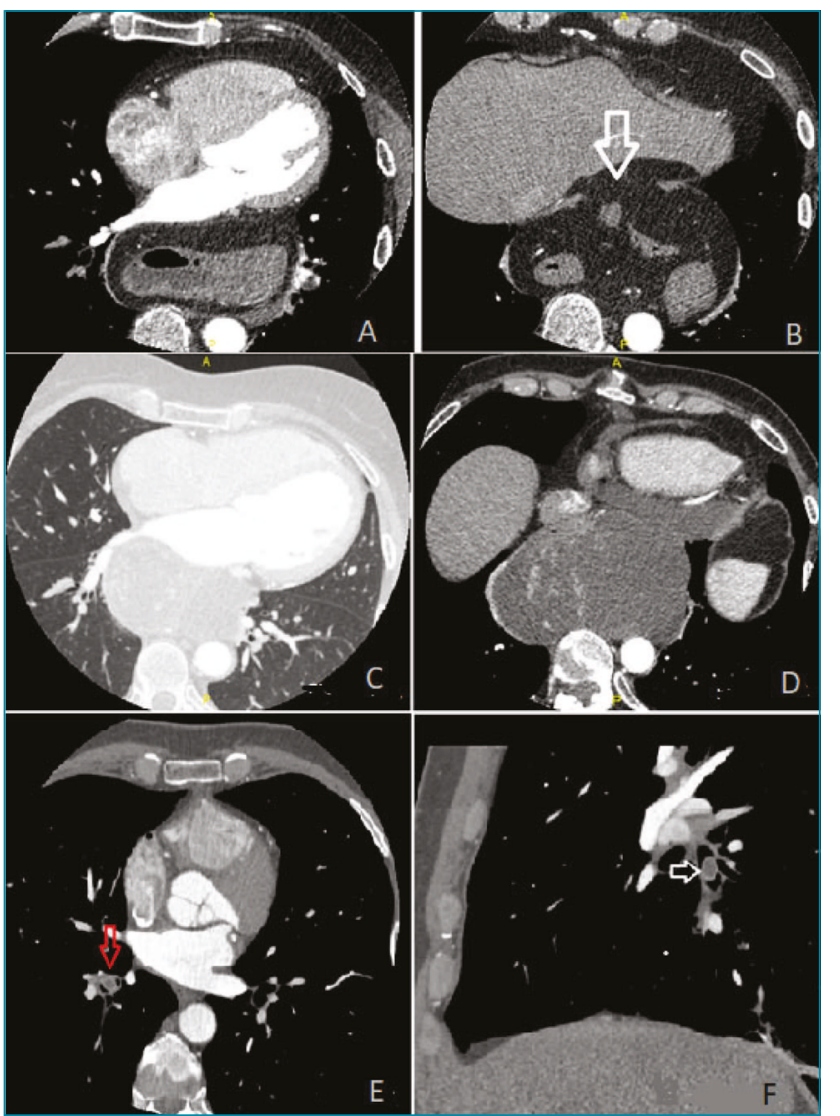

Figure 2. (A) Herniated intra-abdominal soft tissues compressing the left atrium; (B) wide diaphragmatic defect; (C) esophageal mass compressing the left atrium and right pulmonary veins; and (D) giant esophageal mass and pericardial effusion. Foreign body (cherry seed) in right lower lobe bronchus (E) axial section and (F) sagittal section.
Table 1. Comparison of patients with and without pulmonary pathology.

\begin{tabular}{l|c|c|c} 
& $\begin{array}{c}\text { Pulmonary } \\
\text { pathology } \\
(-)(n=784)\end{array}$ & $\begin{array}{c}\text { Pulmonary } \\
\text { pathology } \\
(+)(n=266)\end{array}$ & p-value \\
\hline Age (years) & $51.0 \pm 10.9$ & $55.5 \pm 11.4$ & $<0.001$ \\
\hline Gender (M/F) & $467 / 317$ & $148 / 118$ & 0.261 \\
\hline Smokers (\%) & 43.2 & 60.1 & $<0.001$ \\
\hline
\end{tabular}

n: number; $M / F$ : male/female. The numbers in bold represent the $p$-values statistically significant.

\section{DISCUSSION}

In our study, a total of 274 incidental lung findings were identified in 266 of the 1050 CCTA scans (25.3\%). It was determined that the patients who have incidental lung findings were older and had a higher rate of smoking history. The most common lesion type and localization were (solid) nodules and diffuse distribution (mostly emphysema), respectively. The rate of incidental findings in our study is consistent with previous studies that reported the rate of extracardiac findings at cardiac $\mathrm{CT}$ as 25 and $26.6 \%$ in CCTA ${ }^{9}$. In the study by Yorgun et al. which includes 1,206 subjects, 186 pulmonary abnormalities were detected in 171 patients $(14.1 \%)^{10}$. The mean age of the study population was 58.7 years. In this study, the mean age of the patients was $52.2 \pm 11.2$ years although the possibility of pathological findings has been found to increase significantly over the age of 40.5 years. When compared, the percentage of our incidental findings is higher although the mean age of the study population is lower. 
The discordance may be due to the time elapsed between the two studies and environmental, socioeconomic, and climatic differences. In recent years, with the rapid development of computed tomography technology, the radiation dose has gradually decreased, and the image quality has increased. This low-dose advantage might have paved the way for the use of CCTA for younger patients with atypical and intense subjective complaints as well as for medium-risk patients with stable angina. In addition, the higher proportion of smokers in our study (47.3 versus 38.9\%) may explain the higher incidence of pulmonary pathologies. Since the probability of malignancy of pulmonary nodules increases after the age of 40 years, incidental findings should be more carefully followed over this age ${ }^{11}$.

There is a positive correlation between smoking and the presence of incidental pulmonary findings $(r=0.147, p<0.001)$, in our study population. A study that demonstrates the prevalence of incidental findings by cardiac CT scanning among patients on hemodialysis reports no correlation between the smoking status and the presence of any incidental findings or pulmonary nodules. Smoking was defined as self-reported lifetime exposure of at least 100 cigarettes, which was very few when compared with our study. This supports the idea that incidental findings are less likely to appear in those exposed to small amounts of cigarette smoke. More information is needed to specify the cutoff value for smoking regarding pulmonary abnormalities in asymptomatic smokers ${ }^{12}$.

The most common incidental findings on CCTA scans are pulmonary nodules, which were consistent with previous studies $^{13}$. The percentage of pulmonary nodules reported in CCTA studies ranges from $9.3-19 \%$ for nodules $<1 \mathrm{~cm}$ and $0.6-2.4 \%$ for nodules $>1 \mathrm{~cm}$. In our study, the percentage of nodules was $9.5 \%(100 / 1,050)$ which is consistent with the literature ${ }^{9}$. In a study by Iribarren et al., 81 out of 459 subjects $(18 \%)$ had noncalcified pulmonary nodules in cardiac computed tomography. The lesion disappeared in $35 \%$, decreased or remained stable in $62 \%$, and there was interval growth in $3 \%$ of the participants who were followed up for a 24 -month period. This study has highlighted that reporting noncalcified pulmonary nodules resulted in substantial rescanning that overwhelmingly revealed the resolution or stability of pulmonary nodules ${ }^{14}$. As a limitation, because of the retrospective design of our study, we have reported the incidental findings but did not follow the consequences of the "important" findings.

The radiologists have high levels of familiarity and adherence to guidelines for pulmonary nodule evaluation, but they may overestimate the quality of evidence in support of the recommendations ${ }^{15}$. In addition, the incidental nodules are usually $<6 \mathrm{~mm}$ in size and do not need further follow up ${ }^{16}$ unless they are subsolid nodules (including those with pure ground-glass or part-solid types) close to $6 \mathrm{~mm}$ in size with suspicious morphology or other risk factors ${ }^{17}$. Haller et al. ${ }^{18}$ recommended classifying the incidental findings into major and minor groups. In this way, prevention of over-tracking the clinically insignificant pathologies such as millimetric nodules or congenital variations and elucidating exceptional conditions such as tumors, pulmonary embolism, and foreign bodies can be possible ${ }^{18}$. Onuma et al. reported 319/552 (58\%) of patients with at least one extracardiac findings of which $22.7 \%$ of them were considered "important" ${ }^{19}$. Clinicians need to be aware of the incidental findings as well as the false-positive results and discuss them with patients, alongside the expected benefits of surveillance imaging ${ }^{20}$. From the perspective of CCTA, it would be favorable both for the patients and the clinicians to classify the incidental lung findings in FOV of CCTA according to their clinical significance.

\section{CONCLUSIONS}

Incidental pulmonary findings are common in CCTA, and they are often benign. Hence, the clinician and the patient must take a joint decision to agree to distinguish benign pathologies of no clinical significance from serious lesions, which are quite rare but vital.

\section{AUTHORS' CONTRIBUTIONS}

TE: Conceptualization, Data Curation, Formal Analysis, Writing - original draft, Writing - review \& editing. BYK: Data Curation, Formal Analysis, Writing - original draft, Writing - review \& editing.

\section{REFERENCES}

1. Fihn SD, Gardin JM, Abrams J, Berra K, Blankenship JC, Dallas AP. 2012 ACCF/AHA/ACP/AATS/PCNA/SCAI/ STS guideline for the diagnosis and management of patients with stable ischemic heart disease: a report of the American College of Cardiology Foundation/American Heart Association task force on practice guidelines, and the American College of Physicians, American Association for Thoracic Surgery, Preventive Cardiovascular Nurses Association, Society for Cardiovascular Angiography and Interventions, and Society of Thoracic Surgeons. J Am Coll Cardiol. 2012;60(24):e44-64. https://doi.org/10.1016/j. jacc.2012.07.013 
2. Novellis P, Cominesi SR, Rossetti F, Mondoni M, Gregorc $V$, Veronesi $G$. Lung cancer screening: who pays? Who receives? The European perspectives. Transl Lung Cancer Res. 2021;10(5):2395-406. https://doi.org/10.21037/t|cr-20-677

3. Yoshida M, Utsunomiya D, Inoue T, Nakaura T, Sakaino N, Harada K, et al. Prevalence of extracardiac findings in patients undergoing coronary computed tomography and additional low-dose whole-body computed tomography. Jpn J Radiol. 2020;38(2):144-53. https://doi.org/10.1007/s11604-01900906-3

4. O'Sullivan JW, Muntinga T, Grigg S, loannidis JPA. Prevalence and outcomes of incidental imaging findings: umbrella review. BMJ. 2018;361:k2387. https://doi.org/10.1136/bmj.k2387

5. Alfakih K, Budoff M. Multi-detector computed tomography coronary angiography: the incidental lung findings. J R Soc Med. 2011;104(2):50-1. https://doi.org/10.1258/jrsm.2010.100344

6. Kantarcı M, Duran C, Durur I, Ulusoy L, Gülbaran M, Önbaş Ö. Koroner arterlerin değerlendirilmesinde multidedektör BT Anjiyografi: Teknik, anatomi ve varyasyonlar. Bilgisayarlı Tomografi Bülteni 2004 [cited on Sept. 17, 2020];8(2):90-8. [Multidetector CT angiography in the evaluation of coronary arteries: Technique, anatomy and variations].Available from: http://www.turkmedline.net/detay. $\mathrm{html}$ ?id=af3c937027c0ae7\&language=tr\&mysearchvalue=192\%20 2004\%208\%202

7. Iyama Y, Nakaura T, Kidoh M, Oda S, Utsunomiya D, Sakaino N, et al. Submillisievert radiation dose coronary $\mathrm{CT}$ angiography: clinical impact of the knowledge-based iterative model reconstruction. Acad Radiol. 2016;23(11):1393-401. https:// doi.org/10.1016/j.acra.2016.07.005

8. Kang EJ. Clinical applications of wide-detector CT scanners for cardiothoracic imaging: an update. Korean J Radiol. 2019;20(12):1583-96. https://doi.org/10.3348/kjr.2019.0327

9. Koonce J, Schoepf JU, Nguyen SA, Northam MC, Ravenel JG. Extra-cardiac findings at cardiac CT: experience with 1,764 patients. Eur Radiol. 2009;19(3):570-6. https://doi.org/10.1007/ s00330-008-1195-3

10. Yorgun $H$, Kaya EB, Hazirolan $T$, Ateş AH, Canpolat U, Sunman $\mathrm{H}$, et al. Prevalence of incidental pulmonary findings and early follow-up results in patients undergoing dual-source 64-slice computed tomography coronary angiography. J Comput Assist Tomogr. 2010;34(2):296-301. https://doi.org/10.1097/ RCT.0b013e3181c1d0e4

11. Li C, Liao J, Cheng B, Li J, Liang $H$, Jiang $Y$, et al. Lung cancers and pulmonary nodules detected by computed tomography scan: a population-level analysis of screening cohorts. Ann Transl Med. 2021;9(5):372. https://doi. org/10.21037/atm-20-5210

12. Jaar BG, Zhang L, Chembrovich SV, Sozio SM, Shafi T, Scialla $J$ J, et al. Incidental findings on cardiac computed tomography in incident hemodialysis patients: the predictors of arrhythmic and cardiovascular events in end-stage renal disease (PACE) study. BMC Nephrol. 2014;15:68. https://doi.org/10.1186/14712369-15-68

13. Boldeanu I, Perreault Bishop J, Nepveu S, Stevens LM, Soulez G, Kieser TM, et al. Incidental findings in CT imaging of coronary artery bypass grafts: results from a Canadian multicenter prospective cohort. BMC Res Notes. 2018;11(1):72. https:// doi.org/10.1186/s13104-018-3168-1

14. Iribarren C, Hlatky MA, Chandra M, Fair JM, Rubin GD, Go AS, et al. Incidental pulmonary nodules on cardiac computed tomography: prognosis and use. Am J Med. 2008;121(11):98996. https://doi.org/10.1016/j.amjmed.2008.05.040

15. Gould MK, Altman DE, Creekmur B, Qi L, Bie E, Golden S, et al. Guidelines for the evaluation of pulmonary nodules detected incidentally or by screening: a survey of radiologist awareness, agreement, and adherence from the watch the spot trial. J Am Coll Radiol. 2021;18(4):545-53. https://doi. org/10.1016/j.jacr.2020.10.003

16. Swensen SJ, Jett JR, Hartman TE, Midthun DE, Sloan JA, Sykes AM, et al. Lung cancer screening with CT: Mayo Clinic experience. Radiology. 2003;226(3):756-61. https://doi. org/10.1148/radiol.2263020036

17. MacMahon H, Naidich DP, Goo JM, Lee KS, Leung ANC, Mayo $J R$, et al. Guidelines for management of incidental pulmonary nodules detected on CT images: from the Fleischner Society 2017. Radiology. 2017;284(1):228-43. https://doi.org/10.1148/ radiol.2017161659

18. Haller S, Kaiser C, Buser P, Bongartz G, Bremerich J. Coronary artery imaging with contrast-enhanced MDCT: extracardiac findings. AJR Am J Roentgenol. 2006;187(1):105-10. https:// doi.org/10.2214/AJR.04.1988

19. Onuma Y, Tanabe K, Nakazawa G, Aoki J, Nakajima H, Ibukuro K, et al. Noncardiac findings in cardiac imaging with multidetector computed tomography. J Am Coll Cardiol. 2006;48(2):402-6. https://doi.org/10.1016/j.jacc.2006.04.071

20. Nijhuis AAG, Dieng M, Khanna N, Lord SJ, Dalton J, Menzies $A M$, et al. False-positive results and incidental findings with annual CT or $\mathrm{PET} / \mathrm{CT}$ surveillance in asymptomatic patients with resected stage III melanoma. Ann Surg Oncol. 2019;26(6):1860-8. https://doi.org/10.1245/s10434-019-07311-0 